\title{
Does Intravenous Infusion Influence Diagnostic Ultrasound-Induced Pulmonary Capillary Hemorrhage?
}

Douglas L. Miller, PhD (D), Zhihong Dong, MD, Chunyan Dou, MD, Krishnan Raghavendran, MD

Received August 15, 2017, from the Departments of Radiology (D.L.M., Z.D., C.D.) and Surgery (K.R.), University of Michigan, Ann Arbor, Michigan USA. Manuscript accepted for publication November 6, 2017.

This study was supported by the National Heart Lung and Blood Institute via grant HL116434. The information contained herein does not necessarily reflect the position or policy of the United States government, and no official endorsement should be inferred.

Address correspondence to Douglas $L$. Miller, PhD, Department of Radiology, University of Michigan Health System, 1301 Catherine St, 3240A Medical Sciences Building I, Ann Arbor, MI 48109-5667 USA.

E-mail:douglm@umich.edu

Abbreviations

$I V$, intravenous; MI, mechanical index; $\mathrm{PCH}$, pulmonary capillary hemorrhage; US, ultrasound

doi:10.1002/jum.14555
Objectives - Pulmonary diagnostic ultrasound (US) can induce pulmonary capillary hemorrhage $(\mathrm{PCH})$ in mammals. This singular biological effect of diagnostic US imaging was discovered more than 25 years ago but remains poorly understood. Our objective here was to investigate rapid infusion of intravenous fluids as a possible stressor for capillaries, which might enhance pulmonary diagnostic US-induced $\mathrm{PCH}$.

Methods-Rats were anesthetized with Telazol (Zoetis, Inc, Kalamazoo, MI), which yielded relatively low pulmonary diagnostic US-induced $\mathrm{PCH}$, or Telazol and xylazine, which yielded relatively high pulmonary diagnostic US-induced $\mathrm{PCH}$. Groups of rats were not infused or infused either with normal saline, 10\% mannitol, or $5 \%$ albumin. Rats were scanned in a warmed water bath with B-mode US for 5 minutes with a 7.6-MHz linear array set to different mechanical index values to obtain exposure response information. Pulmonary capillary hemorrhage was observed as comet tail artifacts in the image and measured on the lung surface.

Results-For Telazol anesthesia, all of the PCH results were very low, with no significant differences at the maximum output with an in situ peak rarefactional pressure amplitude of 2.1 MPa (on-screen mechanical index, 0.9). The addition of xylazine to the Telazol anesthetic significantly enhanced the PCH $(P<.001)$ without infusion and likewise for the mannitol and albumin infusion. Saline infusion eliminated this enhancement, with significantly reduced $\mathrm{PCH}$ for Telazol-plusxylazine anesthesia $(P<.001)$; however, both mannitol and albumin infusion resulted in significantly more $\mathrm{PCH}$ than saline infusion $(P<.01)$.

Conclusions-These results show $\mathrm{PCH}$ dependence on the specific intravenous infusion fluid and illustrate the complex importance of physiologic parameters for US-induced PCH.

Key Words - animal studies; basic science (bioeffects); chest/lung; comet tail artifact; crystalloid and colloid infusion; diagnostic ultrasound safety; emergency medicine; mechanical index; pulmonary ultrasound; ultrasound techniques/physics

$\mathrm{D}$ iagnostic ultrasound (US) imaging of mammalian lung can induce pulmonary capillary hemorrhage $(\mathrm{PCH})$. The induction of PCH by pulsed US was discovered more than 25 years ago ${ }^{1}$ but remains poorly understood. Reviews of research have indicated that the phenomenon occurs in mice, rats, and pigs and may be characterized by a threshold for a specific situation. ${ }^{2,3}$ Initially, the lung was expected to receive mostly incidental exposure, such as during echocardiography; therefore, no specific guidance was devised for avoidance of the effect. ${ }^{3}$ At this time, however, direct pulmonary 
diagnostic US has become routine in intensive care, emergency care, and point-of-care medical settings. ${ }^{4-7}$ Pulmonary diagnostic US has been found to be valuable in diagnosis of pneumonia, pulmonary edema, embolism, pneumothorax, atelectasis, diffuse parenchymal disease, respiratory distress syndrome, and lung cancer. ${ }^{8}$ The use of portable US machines allows pulmonary diagnostic US examinations to be performed by the physician at the bedside, essentially replacing the stethoscope. $^{9-11}$ This rapidly expanding use of pulmonary diagnostic US lends some urgency to efforts to define the possible risks of $\mathrm{PCH}$ for patients and to provide suitable safety guidance.

Our research has used actual diagnostic US systems (early work used single-element laboratory systems) to provide relevant information, including pulmonary diagnostic US images, which display the occurrence of $\mathrm{PCH}$ as comet tail artifacts extending from the pleura toward the interior. ${ }^{12}$ The thresholds for this bioeffect appear to have little frequency dependence, ${ }^{13}$ which suggests that the mechanism of acoustic radiation pressure may play a role in the induction of $\mathrm{PCH}^{14}$ Pulmonary capillary hemorrhage has a strong dependence on poorly defined physiologic conditions: the anesthesia methods, such as the use of xylazine, are very important for pulmonary diagnostic US-induced $\mathrm{PCH}$ in rats. ${ }^{15}$ The enhancement phenomenon also occurs for dexmedetomidine, a common clinical sedative that causes little or no respiratory depression. ${ }^{16}$ These findings imply that many patient medical treatments could influence pulmonary diagnostic US-induced $\mathrm{PCH}$ in uncertain ways. Some patient conditions or treatments may worsen the risk, whereas others may alleviate the risk.

The finding that the $\mathrm{PCH}$ effect is low for ketamine and higher for ketamine-plus-xylazine anesthesia ${ }^{15}$ provided a means to test drug treatments and other physiologic conditions for their influence on the bioeffect. For ketamine, conditions leading to enhanced $\mathrm{PCH}$ may be more evident than for ketamine plus xylazine, which already enhances the $\mathrm{PCH}$. Conversely, conditions leading to reduced $\mathrm{PCH}$ may be more evident for ketamine plus xylazine than for ketamine anesthesia, which already has a low effect. Therefore, testing rat models of various clinical conditions with both ketamine and ketamineplus-xylazine anesthesia could readily identify conditions that either enhance or reduce $\mathrm{PCH}$. One problem with this duel strategy is that ketamine, a dissociative agent, ${ }^{17}$ provides poor muscle tone, immobility, and analgesia at normal doses. Telazol (Zoetis, Inc, Kalamazoo, MI), which consists of tiletamine (related to ketamine) plus zolazepam (benzodiazepine-related tranquilizer), is an attractive alternative to ketamine and provides light anesthesia with immobility. In this study, Telazol was compared to Telazol plus xylazine for use as a dual strategy of evaluating physiologic conditions for their influence on pulmonary diagnostic US-induced $\mathrm{PCH}$.

A conceptually simple physiologic condition is the infusion of intravenous (IV) fluids. Rapid infusion of IV fluids, such as saline, can lead to hypervolemia and eventual pulmonary edema, which is identifiable by diagnostic US B-lines. ${ }^{18}$ Hypothetically, fluid infusion leading to vascular hypervolemia and possibly to pulmonary edema may do so by stressing pulmonary capillaries, thus predisposing the lung to PCH. Saline is a crystalloid infusion fluid that rapidly distributes between vascular and tissue interstitial compartments. Another clinical IV fluid is mannitol, a sugar alcohol used at a hypertonic concentration to dehydrate tissues, such as to reduce intracranial pressure due to severe traumatic brain injury owing to its inability to cross the blood-brain barrier. Mannitol also can cause diuresis, possibly reducing edema, such as pulmonary edema. ${ }^{19}$ Clinical IV infusion of an albumin solution can produce increased pulmonary blood volume without potentially increasing pulmonary edema. ${ }^{18}$ Intravenous albumin is a colloid fluid that tends to remain within the vasculature, thus increasing plasma volume. Rapid infusion of very high volumes (eg, 10\% of body weight) of saline, mannitol, or plasma, can lead to a life-threatening fluid overload, which tends to produce pulmonary interstitial edema with saline, alveolar edema with plasma (albumin), and both with hypertonic mannitol. ${ }^{20}$ In this study, pulmonary diagnostic USinduced $\mathrm{PCH}$ variations from infusion of these fluids were assessed by using the strategy of Telazol in comparison to Telazol-plus-xylazine anesthesia.

\section{Materials and Methods}

\section{Animal Preparation}

All in vivo animal procedures were conducted with the approval and guidance of the Institutional Animal Care and Use Committee of the University of Michigan. Female rats (Sprague Dawley; Charles River, Wilmington, MA) were used for this study, as described previously. ${ }^{12}$ Anesthesia was accomplished either with Telazol at $90 \mathrm{mg} / \mathrm{kg}$ only or with Telazol plus xylazine 
(Xylamed injection; Bimeda-MTC Animal Health, Inc, Cambridge, Ontario, Canada) at $9 \mathrm{mg} / \mathrm{kg}$. The use of xylazine for anesthesia was previously shown to enhance pulmonary diagnostic US-induced $\mathrm{PCH}^{15}$ possibly due to an impact on pulmonary capillaries. The use of high doses of xylazine is also associated with induction of pulmonary edema. ${ }^{21,22}$ The right thorax of all rats was shaved and depilated for US transmission. The rats were mounted in a $38^{\circ} \mathrm{C}$ degassed water bath for US exposures of the right lung. This water bath method provides reproducible US coupling and exposure and maintains the body temperature of the rats.

A jugular vein catheter was set for IV injection of fluids in the second part of the study. Three IV fluids were used: normal saline $(0.9 \%$ sodium chloride injection; Hospira, Lake Forest, IL) was the standard medical IV solution administered for fluid replenishment. Mannitol, 10\% (Osmitrol 10\% mannitol injection; Baxter Healthcare, Deerfield, IL), was a hypertonic diuretic that acted to dehydrate tissue with $596 \mathrm{mOsmol}$. Albumin (albumin 5\% solution; CSL Behring, LLC, Kankakee, IL) was given as a volume-expanding colloid with normal osmolarity $(310 \mathrm{mOsmol})$. These fluids were injected rapidly via a syringe pump at $7 \mathrm{ml} / \mathrm{kg} / \mathrm{min}$ for 5 minutes just before US scanning, which was intended to perturb the pulmonary capillaries in these acute tests. The rats were then scanned by diagnostic US for 5 minutes, followed by euthanasia 5 minutes later. This infusion volume totaled $3.5 \%$ of body weight $(\approx 50 \%$ of blood volume). This level of infusion did not produce discernable stress in the rats over the time of the tests, which can occur for higher infusion volumes. In an acute study by Manenti et $\mathrm{al}^{20}$ infusion of $10 \%$ of body weight of these IV fluids resulted in tachypnea and cyanosis, followed by death within 15 minutes.

\section{Ultrasound}

An HDI 5000 diagnostic US machine (Philips Healthcare, Andover, MA) with a CL15-7 linear array was used for B-mode scanning with a $2-\mathrm{cm}$ image depth, a $1-\mathrm{cm}$ focal depth, and 39 frames per second, as described previously. ${ }^{12}$ The transducer was set up in the water bath with an adjustable gantry to aim through an intercostal space at the right cranial or medial lobe of the rat lung. The pleural surface was at a depth of about 5 to $6 \mathrm{~mm}$, with the transducer partially in contact with the skin. The pulses of US had a center frequency of $7.6 \mathrm{MHz}$ with a pulse repetition frequency of $10 \mathrm{kHz}$. The on- screen mechanical index (MI) was set to 0.21 for aiming and then quickly raised to an on-screen MI of $0.37,0.52$, 0.7 , or 0.9 (maximum for this transducer) for 5 minutes of scanning. The acoustic parameters were measured with a calibrated hydrophone as described previously ${ }^{13}$ and are listed in Table 1, including peak rarefactional pressure amplitude, peak mean pressure amplitude, and spatial-peak pulse-average intensity.

\section{Measured End Points}

Measured physiologic parameters included heart rate and oxygen saturation (Physiosuite; Kent Scientific Corp, Torrington, CT). This system allowed measurement of higher heart rates than possible with standard veterinary monitors. The wet/dry weight of the left lobe of the lung was measured by weighing the sample, drying for 24 hours at $37^{\circ} \mathrm{C}$, and reweighing. For the rats with infusion, the hematocrit was measured in baseline samples and in posttest samples. This information was used to estimate the percentage of increase in blood volume accomplished by the infusions as 100 times the difference between the posttest hematocrit and baseline, divided by the baseline result. In addition, a noninvasive blood pressure system (Coda monitor; Kent Scientific Corp) was used on the tail of the rats to measure blood pressure before and after the test.

Exposure-related end points included the percentage of the bright-line image of the lung surface, which was involved with comet tail artifacts at the end of exposure, and measurement of $\mathrm{PCH}$ areas on the lung surface, as described previously. ${ }^{12,13,15,16}$ Briefly, at euthanasia, the trachea was tied off, and the lungs were removed. The right lobes, which were the targets of the imaging, were then examined and photographed by a stereomicroscope with a digital camera (Spot Flex; Diagnostic Instruments, Inc, Sterling Heights, MI). The

Table 1. Measured In Situ Exposure Parameters for the On-screen MI settings Used Here

\begin{tabular}{|c|c|c|c|}
\hline $\begin{array}{l}\text { On-screen } \\
\text { MI }\end{array}$ & $\begin{array}{l}\text { PRPA, } \\
\text { MPa }\end{array}$ & $\begin{array}{l}\text { PMPA, } \\
\text { MPa }\end{array}$ & $\begin{array}{c}I_{\mathrm{SPPA}}, \\
\mathrm{W} \mathrm{cm}^{-2}\end{array}$ \\
\hline 0.9 & $2.13 \pm 0.21$ & $2.77 \pm 0.18$ & $170 \pm 20$ \\
\hline 0.7 & $1.71 \pm 0.19$ & $2.22 \pm 0.16$ & $114 \pm 15$ \\
\hline 0.52 & $1.34 \pm 0.13$ & $1.68 \pm 0.14$ & $75 \pm 13$ \\
\hline 0.37 & $1.02 \pm 0.11$ & $1.22 \pm 0.11$ & $40.5 \pm 7.4$ \\
\hline 0.21 (aiming) & $0.55 \pm 0.06$ & $0.62 \pm 0.06$ & $10.4 \pm 2.0$ \\
\hline
\end{tabular}

Data are presented as mean \pm SD. I ISPA indicates spatial-peak pulse-average intensity; PMPA, peak mean pressure amplitude; and PRPA, peak rarefactional pressure amplitude. 
photographs of the lungs were used to measure the approximate diameter and area of the region of $\mathrm{PCH}$ on the lung surface with image analysis software (Spot version 5.1; Diagnostic Instruments, Inc). The PCH was readily identified as irregular bright red areas along the line of the US scan plane and often the same length as the comet tail artifact length in the image. These were manually outlined by the software with the appropriate scale calibration, which gave the area in square millimeters.

\section{Experimental Plan and Statistics}

The comparison of Telazol-only to Telazol-plus-xylazine (TX) anesthesia was performed first, and the results indicated that the different anesthetics provided a reasonable dual-test strategy. This finding led to performance of the experiments with Telazol only and Telazol plus xylazine for each infusion solution. The two parts were done by the same methods, except for the added hematocrit and blood pressure measurements for the infusion part. Physiologic parameters for the experimental conditions are listed in Table 2. The addition of xylazine for Telazol-plus-xylazine anesthesia gave significant reductions in the heart rate, oxygen saturation, and blood pressure relative to Telazol only, as expected. For each condition, groups of 5 or 6 rats were scanned at the maximum on-screen MI value of 0.9 and additional lower values including on-screen MIs of 0.7, 0.52, and 0.37 . For reference, the guideline upper limit for diagnostic US is an on-screen MI of 1.9, which is substantially higher than available from the small, highresolution transducer. The response for Telazol-only conditions was very low for the infusion tests, and only on-screen MI 0.9 groups were included. One sham Telazol-plus-xylazine group was included, using the on- screen MI for aiming only. A total of 42 rats (7 groups of 6) were included in the Telazol-only versus Telazolplus-xylazine test, and 65 rats were included in the infusion experiment (13 groups of 5). The groups and exposure parameters are presented in Table 3. Statistical comparisons used the $z$ test of proportions for comparison of two groups, with significance assumed at $P \leq .053$ ( $P=.053$ corresponds to $4 / 5$ positive results, compared to $0 / 5$ for shams). The results were unexpectedly mostly negative. Therefore, the lowest on-screen MI group for each Telazol-plus-xylazine condition, which was extended to lower values until no positive effect was found, was considered a sham. Two-way analyses of variance were conducted to compare the importance of the on-screen MI exposure setting relative to the addition of xylazine (Telazol only versus Telazol plus xylazine) and relative to the infusion solutions (saline, mannitol, and albumin). In addition, a linear regression was performed on the exposure response trends of groups including any $\mathrm{PCH}$ detected to estimate thresholds as the 0 -crossing point.

\section{Results}

The general physiologic measurements are listed in Table 2. The differences in the heart rate, oxygen saturation, and blood pressure were all highly significantly different $(P<.01)$ between Telazol-only and Telazol-plusxylazine anesthesia. The mean blood pressure values were variable for the noninvasive method, and there were no significant differences between before and after values. For Telazol wet/dry ratios, there was a significant decrease for saline infusion, no significant difference for mannitol infusion, but a significant increase for albumin infusion. For Telazol plus xylazine, all of the wet/dry

Table 2. Measured Physiologic Parameters for the 4 Different Conditions With and Without Xylazine

\begin{tabular}{|c|c|c|c|c|c|c|c|c|}
\hline \multirow[b]{2}{*}{ Parameter } & \multicolumn{4}{|c|}{ Telazol Only } & \multicolumn{4}{|c|}{ Telazol + Xylazine } \\
\hline & None & Saline & Mannitol & Albumin & None & Saline & Mannitol & Albumin \\
\hline $\mathrm{n}$ & 18 & 5 & 5 & 5 & 24 & 10 & 20 & 20 \\
\hline Weight, g & $255 \pm 9$ & $261 \pm 19$ & $257 \pm 25$ & $262 \pm 26$ & $251 \pm 16$ & $268 \pm 18$ & $253 \pm 16$ & $258 \pm 24$ \\
\hline $\mathrm{HR}$, beats/min & $437 \pm 58$ & $415 \pm 36$ & $410 \pm 48$ & $420 \pm 28$ & $249 \pm 27$ & $295 \pm 39$ & $316 \pm 49$ & $322 \pm 39$ \\
\hline $\mathrm{SpO}_{2}, \%$ & $93 \pm 5$ & $88 \pm 6$ & $84 \pm 9$ & $89 \pm 4$ & $77 \pm 9$ & $79 \pm 12$ & $82 \pm 8$ & $76 \pm 8$ \\
\hline MBP before, $\mathrm{mm} \mathrm{Hg}$ & & $126 \pm 28$ & $115 \pm 19$ & $113 \pm 18$ & & $82 \pm 20$ & $78 \pm 14$ & $94 \pm 28$ \\
\hline MBP after, mm Hg & & $109 \pm 36$ & $96 \pm 18$ & $99 \pm 17$ & & $81 \pm 11$ & $81 \pm 14$ & $78 \pm 15$ \\
\hline Wet/dry & $4.3 \pm 0.2$ & $4.1 \pm 0.1$ & $4.5 \pm 0.6$ & $4.9 \pm 0.3$ & $4.2 \pm 0.2$ & $4.5 \pm 0.3$ & $4.5 \pm 0.3$ & $4.8 \pm 0.4$ \\
\hline BV increase, \% & & $23 \pm 9$ & $31 \pm 7$ & $37 \pm 14$ & & $23 \pm 11$ & $30 \pm 13$ & $34 \pm 13$ \\
\hline
\end{tabular}

Data are presented as mean \pm SD where applicable. BV indicates blood volume; HR, heart rate; MBP, mean blood pressure before and after infusion; and $\mathrm{SpO}_{2}$, oxygen saturation. 
results for the infusions were significantly greater than for no infusion. The hematocrit of about 0.45 was reduced for all 3 infusions by $18 \%$ to $27 \%$, with a statistically significant blood volume increase for albumin infusion relative to no infusion.

The measured end point results are presented in Table 3. The primary measure of the effect was the $\mathrm{PCH}$ area. The comet tail artifacts were also measured and closely tracked the $\mathrm{PCH}$ length on the lungs. For the comparison of Telazol-only and Telazol-plusxylazine anesthesia, the added xylazine yielded a significant increase in $\mathrm{PCH}$ at the on-screen MI of 0.9 $(P<.001)$, and greater observed $\mathrm{PCH}$ at lower onscreen MI values. The analyses of variance showed that the xylazine had a significant impact $(P<.001)$ as did the on-screen MI setting $(P<.001)$; furthermore, the on-screen MI of 0.9 was statistically significantly different from on-screen MIs of $0.37,0.52$, and 0.7. This observation was similar to ketamine compared to ketamine-plus-xylazine results ${ }^{15}$ and indicated that the strategy of Telazol-only versus Telazol-plus-xylazine tests for gauging the influence of different conditions on $\mathrm{PCH}$ sensitivity was plausible.

A comparison between the no-infusion and infusion parts of the study revealed no substantial enhancement due to the infusions, as shown in Figure 1 for the onscreen MI 0.9 setting. All of the infusions for Telazol only led to $\mathrm{PCH}$, which was not significantly different from 0 , apparently reducing the significant $\mathrm{PCH}$ seen for Telazol only without infusion. All of the $\mathrm{PCH}$ results with Telazol plus xylazine were significantly greater $(P<.01)$ than the corresponding Telazol-only condition except for the saline infusion, which was somewhat less $(P=.078)$. However, the saline infusion with Telazol plus xylazine produced a substantial reduction in $\mathrm{PCH}$ relative to the Telazol plus xylazine without infusion $(P<.001)$.

For the infusion part of the study, no significant $\mathrm{PCH}$ was observed for Telazol-only anesthesia (Figure 1). For Telazol-plus-xylazine anesthesia, the $\mathrm{PCH}$ for mannitol or albumin infusion was comparable to the noinfusion result (Figure 1). The PCH for saline infusion

Table 3. Pulmonary Capillary Hemorrhage Results for Diagnostic US Scanning at the Indicated Onscreen MIs

\begin{tabular}{|c|c|c|c|c|c|c|c|}
\hline Condition & $\begin{array}{c}\text { On-screen } \\
\text { MI }\end{array}$ & $\begin{array}{l}\text { US CTA, } \\
\text { mm }\end{array}$ & $\begin{array}{c}\text { CTA, } \\
\%\end{array}$ & $\begin{array}{c}\text { Positive } \\
\text { Proportion }\end{array}$ & $\begin{array}{l}\text { Area, } \\
\mathrm{mm}^{2}\end{array}$ & $\begin{array}{c}\text { Intercept, } \\
\mathrm{MPa}\end{array}$ & $r^{2}$ \\
\hline TO, none & 0.9 & $2.7 \pm 2.9$ & $26 \pm 28$ & $5 / 6$ & $1.3 \pm 1.0^{a}$ & & \\
\hline TO, none & 0.7 & $0.8 \pm 1.3$ & $8.7 \pm 14.7$ & $2 / 6$ & $0.2 \pm 0.3$ & 1.6 & 0.37 \\
\hline TO, none & 0.52 & 0 & 0 & $0 / 6$ & 0 & & \\
\hline TX, none & 0.9 & $8.4 \pm 2.3$ & $87 \pm 24$ & $6 / 6$ & $4.6 \pm 1.3^{a}$ & & \\
\hline TX, none & 0.7 & $2.3 \pm 2.8$ & $36 \pm 30$ & $4 / 6$ & $1.2 \pm 1.9$ & & \\
\hline TX, none & 0.52 & $0.4 \pm 0.7$ & $4.3 \pm 6.8$ & $2 / 6$ & $0.08 \pm 0.14$ & 1.4 & 0.67 \\
\hline TX, none & 0.21 & 0 & 0 & $0 / 6$ & 0 & & \\
\hline TO, saline & 0.9 & $0.2 \pm 0.5$ & $2.0 \pm 4.4$ & $1 / 5$ & $0.37 \pm 0.83$ & ND & \\
\hline TX, saline & 0.9 & $1.7 \pm 1.9$ & $19 \pm 21$ & $3 / 5$ & $0.5 \pm 0.6$ & & \\
\hline TX, saline & 0.7 & $0.2 \pm 0.5$ & $2.4 \pm 5.3$ & $1 / 5$ & $0.01 \pm 0.03$ & 1.7 & 0.33 \\
\hline TO, mannitol & 0.9 & $0.4 \pm 0.8$ & $3.0 \pm 6.7$ & $1 / 5$ & $0.8 \pm 1.8$ & ND & \\
\hline TX, mannitol & 0.9 & $4.4 \pm 3.4$ & $44 \pm 36$ & $4 / 5$ & $4.2 \pm 3.6^{a}$ & & \\
\hline TX, mannitol & 0.7 & $4.5 \pm 2.3$ & $46 \pm 23$ & $5 / 5$ & $2.6 \pm 2.2^{a}$ & & \\
\hline TX, mannitol & 0.52 & $0.9 \pm 1.2$ & $9.9 \pm 14.5$ & $1 / 5$ & $0.3 \pm 0.7$ & 1.3 & 0.33 \\
\hline TX, mannitol & 0.37 & 0 & 0 & $0 / 5$ & 0 & & \\
\hline TO, albumin & 0.9 & $0.4 \pm 1.0$ & $3.8 \pm 4.5$ & $1 / 5$ & $2.1 \pm 4.6$ & ND & \\
\hline TX, albumin & 0.9 & $5.8 \pm 3.1$ & $57 \pm 28$ & $5 / 5$ & $7.3 \pm 4.7^{a}$ & & \\
\hline TX, albumin & 0.7 & $2.5 \pm 3.4$ & $25 \pm 31$ & $3 / 5$ & $3.1 \pm 4.0$ & & \\
\hline TX, albumin & 0.52 & $1.0 \pm 1.7$ & $9.5 \pm 15.4$ & $2 / 5$ & $1.3 \pm 2.1$ & 1.2 & 0.45 \\
\hline TX, albumin & 0.37 & 0 & 0 & $0 / 5$ & 0 & & \\
\hline
\end{tabular}

Data are presented as mean \pm SD where applicable. CTA, \% indicates percentage of comet tail artifacts in the bright-line lung surface image; TO, Telazol only; TX, Telazol plus xylazine; and US CTA, US image comet tail artifact width. The intercept for the linear regression on the means (see Figure 2) approximates the threshold peak rarefactional pressure amplitude for the condition with the corresponding coefficient of determination $\left(r^{2}\right)$. The linear regression intercepts are the apparent thresholds for $\mathrm{PCH}$; regressions were not determined (ND) for the single data.

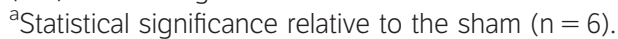


was very low and not significantly different from 0 even for Telazol plus xylazine. The analysis of variance indicated that both mannitol and albumin infusion with Telazol plus xylazine gave relatively large pulmonary diagnostic US-induced PCHs, which were significantly greater than the result for saline infusion $(P<.005$ for mannitol; $P<.001$ for albumin). Furthermore, the results for both the on-screen MI of $0.9(P<.001)$, and $0.7(P=.015)$ results were significantly different from that for the on-screen MI of 0.37 (taken as the sham).

Threshold determination by linear regression provides an important measure of sensitivity, which uses all of the data points. For the Telazol-only infusion conditions with only 1 point (on-screen MI of 0.9; Table 3), the threshold could not be determined by linear regression but may be assumed to be approximately equal to the maximum exposure peak rarefactional pressure amplitude of 2.1 MPa. The data sets for determinations of thresholds with Telazol plus xylazine were small, as shown in Figure 2, and the linear regressions had modest coefficients of determination $\left(r^{2}\right.$; Table 3). For the Telazol plus xylazine without infusion and with mannitol and albumin infusions, the 0 intercepts for linear regression were all about the same, ranging from peak rarefactional

Figure 1. Pulmonary capillary hemorrhage areas measured on the lung surface for the on-screen MI 0.9 groups. Asterisks signify means that are statistically significantly different from the sham. The fraction above each bar is the positive fraction for the group. The results for saline infusion were remarkably low for both the Telazol-only and Telazol-plus-xylazine anesthesia, which was significantly less than the Telazol-plus-xylazine group with no infusion $(P<.001)$. For Telazol plus xylazine, the no-infusion (none), mannitol infusion, and albumin infusion results were all about the same. However, the mannitol and albumin infusions significantly enhanced the $\mathrm{PCH}$ relative to saline infusion.

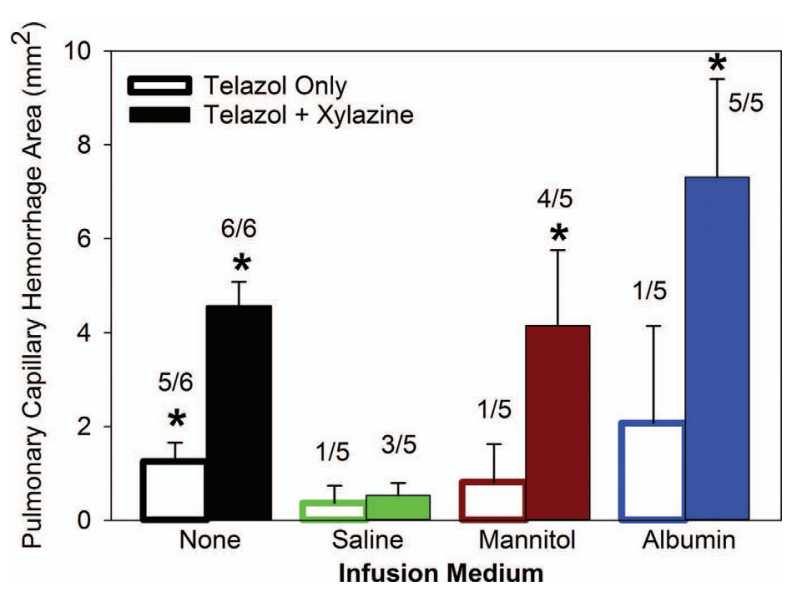

pressure amplitudes of 1.2 to $1.4 \mathrm{MPa}$ (Table 3). For Telazol only with no infusion and Telazol plus xylazine with saline infusion, the 0 crossings were 1.6 to $1.7 \mathrm{MPa}$, about 1 exposure step higher than those for the other conditions.

\section{Discussion}

One goal of this study was to compare pulmonary diagnostic US-induced PCH results obtained with Telazolonly and Telazol-plus-xylazine anesthesia as a means to evaluate enhancement or reduction of the $\mathrm{PCH}$ bioeffect by physiologic conditions. The $\mathrm{PCH}$ effect appeared to be somewhat inhibited relative to previous observations with this US machine and transducer using ketamine ${ }^{12}$ rather than Telazol. The on-screen MI of 0.52 yielded statistically significant $\mathrm{PCH}$ for ketamine and xylazine anesthesia in that study but not in this study with Telazol-plus-xylazine anesthesia. However, the Telazolonly condition produced very similar results as ketamine in a previous study. ${ }^{15}$ The Telazol mixture provides much better immobilization than ketamine only and analgesia sufficient for minor surgical procedures, such as placement of a jugular vein catheter.

The Telazol-only and Telazol-plus-xylazine comparison was used to test for $\mathrm{PCH}$ reduction or enhancement by IV fluid infusion. The main finding in this study was that infusion tended to reduce $\mathrm{PCH}$. The Telazol-

Figure 2. Results for the pulmonary capillary hemorrhage areas for the exposure response tests. TO indicates Telazol-only anesthesia; and TX, Telazol-plus-xylazine anesthesia. The 0 -crossing values of the linear regressions provide values for the $\mathrm{PCH}$ thresholds (see Table 3). The saline infusion eliminated the enhancement effect of the xylazine

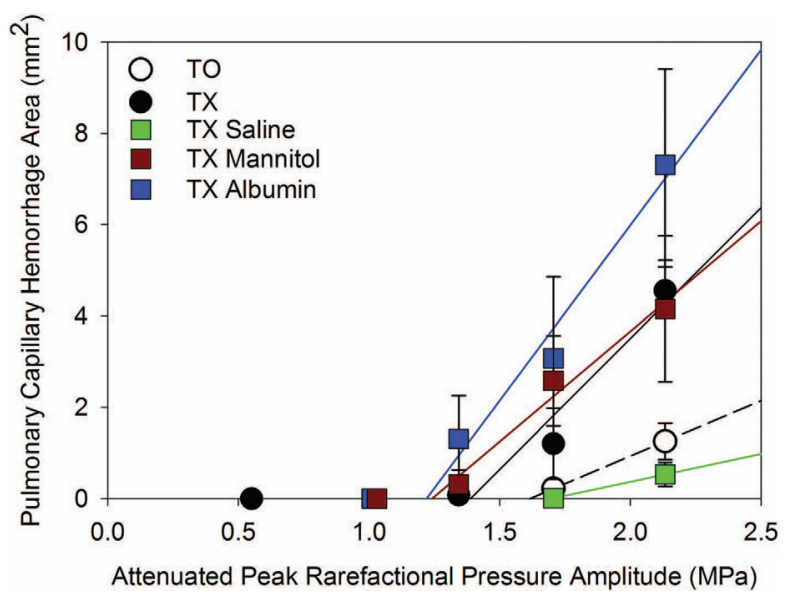


only anesthesia without infusion gave statistically significant PCH $(P<.02)$ at an on-screen MI of 0.9, but Telazol-only conditions with infusion produced no significant $\mathrm{PCH}$. An overall comparison of the $5 / 6$ proportion of occurrence for Telazol only without infusion with the $3 / 15$ occurrence for Telazol only with any infusion was a significant reduction at $P=.028$. For Telazol-plus-xylazine anesthesia, saline infusion eliminated the enhancement by xylazine. The mannitol and albumin infusions had no clear impact on the $\mathrm{PCH}$ relative to the no-infusion part of the study. However, within the infusion part of the study, the comparison of saline to albumin infusion was striking: Saline infusion with Telazol plus xylazine did not give statistically significant $\mathrm{PCH}$ at the on-screen MI of 0.9. However, mannitol and albumin infusion resulted in observed $\mathrm{PCH}$, which was significantly greater $(P<.001)$ than for saline infusion.

In conclusion, the Telazol-only and Telazol-plusxylazine strategy for assessing conditions favoring or inhibiting pulmonary diagnostic US-induced $\mathrm{PCH}$ was validated, with lower $\mathrm{PCH}$ for Telazol only and higher $\mathrm{PCH}$ for Telazol plus xylazine. For infusion, the most substantial change was the elimination of significant pulmonary diagnostic US-induced $\mathrm{PCH}$ for saline infusion with Telazol plus xylazine and the reduction to insignificance of PCH by all the infusions for Telazol-only anesthesia. The mechanism for the reduction in the $\mathrm{PCH}$ effect by saline infusion is uncertain. Because it is neutral with regard to tonicity and subject to redistribution outside the vasculature, the rapid infusion of this crystalloid gave the lowest increase in blood volume. The striking difference between the results for saline and albumin infusion $(P<.001)$ probably arose from the different disposition of crystalloid and colloid infusions. Albumin and mannitol have a much greater tendency than saline to remain within the vasculature, increasing plasma volume and potentially provoking distension and stress, as well as recruitment, of pulmonary capillaries, which could increase vulnerability to pulmonary diagnostic USinduced PCH. Using the Telazol-only and Telazol-plusxylazine test strategy, the infusion did not increase $\mathrm{PCH}$ for Telazol only but rather decreased it. For Telazol plus xylazine, albumin and mannitol had little effect, but saline reduced pulmonary diagnostic US-induced PCH to a level even lower that the Telazol-only result. Overall, the hypothesis that rapid infusion of IV fluids could serve as a possible stressor for pulmonary capillaries, which might enhance pulmonary diagnostic US-induced $\mathrm{PCH}$, was not supported.

\section{References}

1. Child SZ, Hart man CL, Schery LA, Carstensen EL. Lung damage from exposure to pulsed ultrasound. Ultrasound Med Biol 1990; 16: 817-825.

2. American Institute of Ultrasound in Medicine. Section 4: bioeffects in tissues with gas bodies. J Ultrasound Med 2000; 19:97-108, 154-168.

3. Church CC, Carstensen EL, Nyborg WL, Carson PL, Frizzell LA, Bailey MR. The risk of exposure to diagnostic ultrasound in postnatal subjects: nonthermal mechanisms. J Ultrasound Med 2008; 27:565-592.

4. Volpicelli G. Lung sonography. J Ultrasound Med 2013; 32:165-171.

5. Lichtenstein D. Lung ultrasound in the critically ill. Curr Opin Crit Care 2014; 20:315-322.

6. Ahmad S, Eisen LA. Lung ultrasound: the basics. In: Lumb P, Karakitsos D (eds). Critical Care Ultrasound. Philadelphia, PA: Elsevier; 2015 : 106-110.

7. Dietrich CF, Goudie A, Chiorean L, et al. Point of care ultrasound: a WFUMB position paper. Ultrasound Med Biol 2017; 43:49-58.

8. Sartori S, Tombesi P. Emerging roles for transthoracic ultrasonography in pleuropulmonary pathology. World J Radiol 2010; 2:83-90.

9. Lumb P, Karakitsos D (eds). Section VI: general chest ultrasound. In: Lumb P, Karakitsos D (eds). Critical Care Ultrasound. Philadelphia, PA: Elsevier; 2015: 105-137.

10. Irwin Z, Cook JO. Advances in point-of-care thoracic ultrasound Emerg Med Clin North Am 2016; 34:151-157.

11. Sekiguchi $\mathrm{H}$. Tools of the trade: point-of-care ultrasonography as a stethoscope. Semin Respir Crit Care Med 2016; 37:68-87.

12. Miller DL. Induction of pulmonary hemorrhage in rats during diagnostic ultrasound. Ultrasound Med Biol 2012; 38:1476-1482.

13. Miller DL, Dou C, Raghavendran K. The dependence of thresholds for pulmonary capillary hemorrhage on diagnostic ultrasound frequency. Ultrasound Med Biol 2015; 41:1640-1650.

14. Miller DL. Mechanisms for induction of pulmonary capillary hemorrhage by diagnostic ultrasound: review and consideration of acoustical radiation surface pressure. Ultrasound Med Biol 2016; 42:2743-2757.

15. Miller DL, Dou C, Raghavendran K. Anesthetic techniques influence the induction of pulmonary capillary hemorrhage during diagnostic ultrasound in rats. J Ultrasound Med 2015; 34:289-297.

16. Miller DL, Dou C, Dong Z, Raghavendran K. The influence of dexmedetomidine on ultrasound-induced pulmonary capillary hemorrhage in rats. Ultrasound Med Biol 2016; 42:964-970.

17. Flecknel P. Laboratory Animal Anaesthesia. London, England: Academic Press; 2009.

18. Bihari S, Wiersema UF, Schembri D, et al. Bolus intravenous $0.9 \%$ saline, but not $4 \%$ albumin or $5 \%$ glucose, causes interstitial pulmonary edema in healthy subjects. J Appl Physiol (1985) 2015; 119:783792. 
Miller et al—IV Infusion and Ultrasound-Induced Pulmonary Capillary Hemorrhage

19. Teke Z, Adali F, Kelten EC, et al. Mannitol attenuates acute lung injury induced by infrarenal aortic occlusion-reperfusion in rats. Surg Today 2011; 41:955-965.

20. Manenti A, Botticelli A, Buttazzi A, Gibertini G. Acute pulmonary edema after over-infusion of crystalloids versus plasma: histological observations in the rat. Pathologica 1992; 84:331-334.
21. Amouzadeh HR, Sangiah S, Qualls CW Jr, Cowell RL, Mauromoustakos A. Xylazine-induced pulmonary edema in rats. Toxicol Appl Pharmacol 1991; 108:417-427.

22. Amouzadeh HR, Qualls CW Jr, Wyckoff JH III, et al. Biochemical and morphological alterations in xylazine-induced pulmonary edema. Toxicol Pathol 1993; 21:562-571. 